\title{
EMG ACTIVITY OF TRANSVERSUS ABDOMINIS, MULTIFIDUS AND CO-CONTRACTION INDEX IN DIFFERENT PHASES AMONGST VARIED LEVEL ARCHERS: A CROSS-SECTIONAL STUDY
}

\author{
SHEIKH AZHAR', SAURABH SHARMA², SHALINI SHARMA1, TARUSHI TANWAR ${ }^{1}$ \\ IJamia Millia Islamia University, Centre for Physiotherapy and Rehabilitation Sciences, New Delhi, India \\ 2ENRICH Physio Clinic, Melbourne, Australia
}

\begin{abstract}
Mailing address: Saurabh Sharma, Jamia Millia Islamia University, Centre for Physiotherapy and Rehabilitation Sciences, Ghaffar Manzil Colony, Jamia Nagar, Okhla, New Delhi, Delhi 110025, India, e-mail: ssharma@jmi.ac.in
\end{abstract}

\begin{abstract}
Introduction. Core muscle activity is a necessary requirement in most sports. However, its role in archers has not been studied. Therefore, this study sought to determine muscle activity of Transversus abdominis (TA) and Multifidus (MF) and co-contraction index (CCI) using surface electromyography (s EMG) during different phases of shots in archers with different levels of training. Material and Methods. It was an observational study with a cross-sectional design. Twenty-eight healthy male archers were recruited and grouped according to their level of training: Group A (Beginners $(n=9))$, Group B (Trained ( $n=10)$ ), Group C (Elite $(\mathrm{n}=9)$ ). The muscle activation $(\% \mathrm{MVIC})$ for MF and TA for 10 successive archery shots (distance: 10 meters in 200 seconds) was recorded using s EMG for all the phases of the shots along with CCI. Results. Three-way ANOVA revealed a significant difference for TA activity within groups ( $\mathrm{p} \leq 0.001)$ and phases $(\mathrm{p}=0.002)$, A significant difference for MF activity was found within groups $(\mathrm{p} \leq 0.001)$. A significant difference for CCI was found between groups $(\mathrm{p} \leq 0.001)$ and in the interaction between phase $\times$ shot $(\mathrm{p}=0.001)$. Conclusions. The findings of the study showed that elite archers had more activity of the core muscles (TA and MF) and their CCI was found to be much higher with respect to the trained and beginner archers. Therefore, it is essential to incorporate dynamic stabilization exercises into archery training.
\end{abstract}

Key words: paraspinal muscles, muscle contraction, electromyography, sports, athletes

\section{Introduction}

Archery is one of the important Olympic sports and gradually the number of athletes and nations participating in events is increasing. Archery is a sport which requires complex coordinated activity of the muscles for accurate execution of the archery shot [1]. Athletic movements are results of an integrated sequential activation of different muscle groups in the human body's kinetic chain. Trunk core muscles like transversus abdominis (TA) and multifidus (MF) are important due to the pattern of movement involved in the execution of archery shot. Effective and efficient sports performance is largely dependent on the core musculature which happens to be the centre of this kinetic chain providing proximal stability which is essential for distal mobility [2]. Core muscles play an important role in the stabilization of the spine which is essential for appendicular movements of the extremities, which is a necessary for better performance. It has been seen that with a $20 \%$ decrease in the kinetic energy generated from the core, there occurs a $35 \%$ increase in rotational velocity at the shoulder in baseball players, while during a tennis serve, $54 \%$ of the total force production takes place in the lower extremity and the trunk musculature [3]. Similar results have been shown for activities such as kicking and javelin throwing.

Being an Olympic level sport, archery has its own physiological and psychological prerequisite coordination. Neuromuscular conditioning, reaction-timing, attention span, and mental aptitude are noteworthy among others. The skill in this sport is not just about accuracy of the shot, but it is more about the consistency of these shots. One of the studies proposed a procedure for evaluating factors determining and affecting elite performance deduced from multifactorial analysis through a set of physical fitness parameters with the major facets for a perfect technique being constant draw length, constant line of force, balanced control, and economy of effort [4].

Many surface electromyography (s EMG) studies on forearm muscles, the trapezius, biceps and triceps muscles have precisely explained the mechanism of shot and its different phases with quantitative analysis in relation to differences in performance and skill level $[5,6,7,8,9]$, and showed the role and importance of deltoid in shooting which provides dynamic stability to the shoulder complex. Previous research studied the contraction strategy of forearm muscles during all phases of archery and reported significant differences among elite, trained and beginners' reaction timing [5]. Also, swifter action was correlated with the EMG findings. The technique used by archers in different forms (recurve/compound) does not alter the use of proximal muscles and this had been advocated as guiding principles for improving performance and decreasing the chances of injuries identified that the key physical fitness variables are correlated positively with performance in this sport which included upper limb strength, endurance, core muscle strength and flexibility [10]. The hypothesis that can be derived from these findings is that core muscles activity might also play an important role in archery shots.

But to the best of our knowledge, there is a dearth of evidence in the field of archery in which EMG activity of the core muscles (TA, MF) and co-contraction index (CCI) has been examined. Therefore, work over this grey area is needed to better understand the mechanics and muscular activity during different phases of this sport. Athletes of different levels of training and skills were included to understand the underlying effect of training in archery and its force production from the core. The main aim of the present study was to assess the role of core muscles TA and MF and co-contraction index (CCI) using s EMG during different phases of archery shot in three varied groups 
of archers (elite, trained and beginners) with different levels of expertise and training.

\section{Material and Methods}

It was an observational study with a cross-sectional study design. A sample size of twenty-eight healthy male archers with the alpha level of 0.05 and power of 0.80 was analysed by use of statistical power program G. power 3.192. Archers were recruited from JN Stadium, New Delhi, India based on the inclusion criteria as follows: (a) age between 18-28 years, (b) male archers, (c) $\geq 3$ years of archery training under professional guidance and previous participation in international or national level competitions for elite group participants, (d) 1.5-3 years of archery training and having participated in level competitions for trained group participants, (e) $<6$ months of training for beginner group participants. The participants were excluded from the study if they suffered from low back pain (mechanical/non-mechanical) for the last 6 months, radiating pain in the upper extremities or lower extremities, any kind of surgical intervention in the upper extremity or the spine. Prior to participation, all research procedures were explained to the participants and a written informed consent was obtained as per the Declaration of Helsinki, after obtaining clearance from the Institutional Ethics Committee (IEC No.: 169/9/124/JMI/IEC/17) of the university.

This study was conducted at the Centre for Physiotherapy and Rehabilitation Sciences, JMI, New Delhi, India. Archers found eligible as per the inclusion and exclusion criteria were grouped according to their level of training in the sport: Group A (Beginners $(\mathrm{n}=9))$, Group B (Trained $(\mathrm{n}=10)$ ), Group C (Elite $(\mathrm{n}=9))$ following which, a brief explanation was given to them about the purpose and procedure of the study. Before the beginning of the procedure, all participants were assessed for core muscle activity using global stabilization testing (side bridge test: 97 seconds, abdominal test: 136 seconds, back extensor endurance: 160 seconds) [11]. The demographic data (age, height, weight, body mass index (BMI)) was collected as shown in table 1 . Height was measured with the help of a stadiometer, while weight was measured by a digital weighing machine. BMI was calculated using the standard formula (weight in kilograms divided by height in meters squared).

The skin was prepared for s EMG recording by shaving excess hair followed by alcohol swab rubbing to reduce impedance. Bipolar disposable, rectangular self-adhesives EMG electrodes (Medicos Electrodes) and dimensions of $4.4 \mathrm{~cm} \times 1.2 \mathrm{~cm}$ were used which were attached parallel to the muscle fibre orientation, unilaterally to the side which was contralateral to the bow arm over the concerned muscles.

Electrode placement for the TA muscle was done at $2 \mathrm{~cm}$ anteromedial from the anterior superior iliac spine keeping in mind the instructions established in literature, and for lumbar MF it was done lateral to the midline of the body, above and below the line connecting both posterior superior iliac spines [12]. The inter-electrode spacing was kept at $2.5 \mathrm{~cm}$ for the recording electrodes [13]. The ground electrode was placed over superior aspect of the iliac crest of the same side. The muscle activity term is synonymous with \% Maximum Voluntary Isometric Contraction (MVIC). To calculate \% MVIC, first the MVIC was recorded with the help of Power lab 15T (AD Instruments, Australia) for TA muscle which involved evaluation in a sitting position with the archers performing an expiratory manoeuvre with maximal effort followed by abdominal hollowing, while the MF muscle activity was obtained in a prone position and the examiner applied manual resistance to the posterior aspect of scapula of the archer, with the legs strapped or manually stabilized to the table [14]. Afterwards, the participants were asked to perform trunk extension with maximal effort against the resistance [15]. The MVIC trials ensured a maximum effort held for five seconds with an inter-attempt rest interval of 60 seconds. Archers were asked about each trial performance and asked to judge their maximum effort relative to the movement. The trial was repeated if the archer was not satisfied with his effort. A 5-second isometric phase was utilised for the recording purpose. Three trials were recorded and mean value of the three trials was used for normalization of s EMG data. Following this, archers were asked to perform their shot while being recorded. This constituted the root mean square (RMS) activity. Before initiating the archery procedure, archers were given a 10-minute warm-up program, after which they were given a demonstration and explained about the procedure in brief. Electrodes were applied and the $\%$ MVIC values for 10 successive shots at a distance of 10 meters in 200 seconds were recorded. This is the standardized protocol in international archery events. Starting from the pre-draw phase and ending at the follow through phase, the commands were checked to be appropriate with the duration of the shots. The stance was kept as a static entity which the archer held till the completion of the shots. The commands were given keeping in mind the different phases of the shot: a) Pre-draw: The initiation of the shot where both hands were raised and levelled to point at the target; b) Draw: The bow hand remained static and maximum movement was seen in the other arm which underwent drawing (pulling of the string) movement; c) Anchor: The least activity phase where the touch points of the body came in contact with the string and the aiming started; d) Release: The execution of the shot which gave a short burst of huge energy which was contained during the previous phases; e) Follow through: The recovery and feedback feel of the shot which gave an insight subjective understanding and prepared for the future shots. Mean normalized muscle activation was determined by dividing the obtained RMS values with the MVIC reading. The formula used was: \% MVIC = (Muscle activity $($ RMS $) /$ MVIC of the muscle $\times 100$ [16]. Co-contraction index (CCI) is the unified synchronous activation of agonist and antagonist muscles to provide stability to a movement. Every activity requires some amount of co-activation. It is the level of intensity beyond what is necessary to accomplish the activity [17]. CCI was determined using the equation derived by formula which is as follows: EMGS/EMGL* (EMGS + EMGL), where EMGS is the level of activity in a less active muscle and EMGL is the level of activity in a more active muscle [18].

\section{Data Analysis}

The data analysis was performed with the help of SPSS 21.0 version for windows software (SPSS Inc. Chicago, IL, USA). Normality of the data was analysed with Shapiro-Wilk test. Mixed three-way ANOVA was used considering shot as the within-subject factor, while group and phase were taken as between-subject factors to find out group effects, phase effects and shot effects. Group $\times$ phase, group $\times$ shot and phase $\times$ shot interaction were evaluated, respectively. A p value of $<0.05$ was considered significant.

\section{Results}

The beginner, trained and elite archers were $19.56 \pm 1.13$, $20.6 \pm 1.17$ and $22.11 \pm 1.36$ years of age, respectively, while BMI was $21.07 \pm 2.63,21.72 \pm 1.84$ and $22.23 \pm 2.32$, respectively (Tab. 1). The s EMG amplitudes of all the groups were recorded 
for TA and MF during every phase within the ten shots, which was then averaged individually with respective MVIC values resulting in \% MVIC. After analysing the respective data of TA and MF, the CCI of each phase was calculated (Tab. 2).

The three-way ANOVA with repeated measures for TA found a significant difference within groups ( $p \leq 0.001)$ and phases ( $\mathrm{p}=0.002)$; however, no significant difference was noted within shots $(\mathrm{p}=0.809)$, group $\times$ phase $(\mathrm{p}=0.941)$, group $\times$ shot $(\mathrm{p}=0.184)$, and phase $\times$ shot interactions $(\mathrm{p}=0.472)$ (Tab. 3). The three-way ANOVA with repeated measures for MF found a significant difference within groups ( $p \leq 0.001$ ); however, no significant difference was noted within phases $(\mathrm{p}=0.649)$, shots $(\mathrm{p}=0.542)$, group $\times$ phase $(\mathrm{p}=0.855)$, group $\times \operatorname{shot}(p=0.031)$, phase $\times$ shot $(p=0.227)($ Tab. 3$)$. For the CCI, a significant difference was found between groups $(\mathrm{p} \leq 0.001)$ and in the interaction between phase $\times \operatorname{shot}(\mathrm{p}=0.001)$; however, no significant difference was noted within phases $(p=0.252)$, shots $(\mathrm{p}=0.742)$, group $\times$ phase $(\mathrm{p}=0.936)$, group $\times$ shot $(\mathrm{p}=$ $0.08)$, phase $\times$ shot $(\mathrm{p}=0.001)$ (Tab. 3$)$. The post-hoc analysis between group analyses revealed significant differences be-

Table 1. Demographic data of the archers

\begin{tabular}{|c|c|c|c|}
\hline Variables & $\begin{array}{c}\text { Group A } \\
\text { (Beginner) }\end{array}$ & $\begin{array}{c}\text { Group B } \\
\text { (Trained) }\end{array}$ & $\begin{array}{c}\text { Group C } \\
\text { (Elite) }\end{array}$ \\
\hline Age $(\mathrm{yrs})$ & $19.56 \pm 1.13$ & $20.6 \pm 1.17$ & $22.11 \pm 1.36$ \\
\hline Height $(\mathrm{m})$ & $170.89 \pm 5.41$ & $173.2 \pm 4.82$ & $173.33 \pm 6.2$ \\
\hline Weight $(\mathrm{kg})$ & $61.78 \pm 10.14$ & $65.20 \pm 6.25$ & $66.89 \pm 8.29$ \\
\hline BMl & $21.07 \pm 2.63$ & $21.72 \pm 1.84$ & $22.23 \pm 2.32$ \\
\hline
\end{tabular}

* - data is in form of MEAN \pm SD.

Table 2. Muscle activity (\%MVIC) of TA, MF and CCI during different phases of archery

\begin{tabular}{|c|c|c|c|}
\hline Variables & $\begin{array}{c}\text { Group A } \\
\text { (Beginner) }\end{array}$ & $\begin{array}{c}\text { Group B } \\
\text { (Trained) }\end{array}$ & $\begin{array}{c}\text { Group C } \\
\text { (Elite) }\end{array}$ \\
\hline TA Pre-draw & $7.42 \pm 3.35$ & $18.54 \pm 6.67$ & $28.96 \pm 9.78$ \\
\hline TA Draw & $8.48 \pm 4.53$ & $20.08 \pm 6.00$ & $29.89 \pm 9.49$ \\
\hline TA Anchor & $7.54 \pm 5.32$ & $19.59 \pm 5.51$ & $22.90 \pm 8.14$ \\
\hline TA Release & $12.15 \pm 6.76$ & $23.89 \pm 8.03$ & $32.85 \pm 13.88$ \\
\hline $\begin{array}{c}\text { TA Follow } \\
\text { Through }\end{array}$ & $8.75 \pm 3.83$ & $19.89 \pm 4.65$ & $30.02 \pm 9.45$ \\
\hline MF Pre-draw & $7.76 \pm 4.23$ & $14.62 \pm 6.81$ & $17.70 \pm 8.09$ \\
\hline MF Draw & $7.65 \pm 4.20$ & $16.48 \pm 7.92$ & $17.64 \pm 6.82$ \\
\hline MF Anchor & $6.46 \pm 3.15$ & $13.24 \pm 6.19$ & $14.38 \pm 6.11$ \\
\hline MF Release & $8.63 \pm 4.28$ & $13.28 \pm 6.31$ & $15.46 \pm 9.47$ \\
\hline $\begin{array}{c}\text { MF Follow } \\
\text { Through }\end{array}$ & $6.87 \pm 3.50$ & $11.36 \pm 5.16$ & $18.82 \pm 11.45$ \\
\hline CCI Pre-draw & $10.05 \pm 4.53$ & $22.09 \pm 10.06$ & $26.24 \pm 10.32$ \\
\hline CCI Draw & $12.58 \pm 6.72$ & $25.94 \pm 13.01$ & $27.500 \pm 11.56$ \\
\hline CCI Anchor & $8.19 \pm 4.74$ & $17.53 \pm 10.95$ & $21.67 \pm 8.63$ \\
\hline CCI Release & $14.65 \pm 8.49$ & $21.59 \pm 11.84$ & $22.26 \pm 13.14$ \\
\hline $\begin{array}{c}\text { CCI Follow } \\
\text { Through }\end{array}$ & $10.42 \pm 4.36$ & $19.00 \pm 10.18$ & $25.88 \pm 15.18$ \\
\hline
\end{tabular}

* - data is in form of MEAN \pm SD; units = millivolts; CCI: Co-contraction index; MF: Multifidus; MVIC: Maximum Voluntary Isometric Contraction; TA: Transverse Abdominis. tween group A vs group B in TA activity ( $\mathrm{p} \leq 0.001)$, MF activity $(\mathrm{p} \leq 0.001)$ and CCI ( $\mathrm{p} \leq 0.001)$ indicating differences between beginners and trained archers in TA and MF activity and CCI. A significant difference was found between group B vs group $C$ for TA activity ( $\mathrm{p} \leq 0.001$ ), while a non-significant difference was found in MF activity $(\mathrm{p} \leq 0.001)$ and CCI $(\mathrm{p}=0.294)$ indicating differences between trained and elite archers only in TA. There was a significant difference between group $A$ vs group $C$ in TA activity ( $\mathrm{p} \leq 0.001)$, MF activity $(\mathrm{p} \leq 0.001)$ and CCI $(\mathrm{p} \leq 0.001)$ indicating differences between beginner and elite archers in TA activity, MF activity and CCI activity (Tab. 4). The post-hoc analysis between phase analyses found a significant difference between Anchor vs Release in TA activity only ( $\mathrm{p}=0.001)($ Tab. 4).

Table 3. Summary of repeated measures of ANOVA for TA, MF and $\mathrm{CCI}$

\begin{tabular}{|c|c|c|c|c|}
\hline Variables & Df & F-value & p-value & $\begin{array}{c}\text { Partial eta } \\
\text { Square }\end{array}$ \\
\hline TA Group & 2 & 80.645 & $\leq 0.001^{*}$ & 0.563 \\
\hline TA Phase & 4 & 4.408 & $0.002^{*}$ & 0.124 \\
\hline TA Shot & 6.887 & 0.531 & 0.809 & $0.004^{*}$ \\
\hline TA Group $\times$ Phase & 8 & 200.079 & 0.941 & $0.0022^{*}$ \\
\hline TA Group $\times$ Shot & 13.775 & 1.331 & 0.184 & $0.021^{*}$ \\
\hline TA Phase $\times$ Shot & 27.550 & 0.996 & 0.472 & $0.031^{*}$ \\
\hline MF Group & 2 & 23.240 & $\leq 0.001^{*}$ & 0.271 \\
\hline MF Phase & 4 & 0.621 & 0.649 & $0.019^{*}$ \\
\hline MF Shot & 6.534 & 0.847 & 0.542 & $0.007^{*}$ \\
\hline MF Group $\times$ Phase & 8 & 219.790 & 0.855 & $0.031^{*}$ \\
\hline MF Group $\times$ Shot & 13.068 & 1.863 & 0.311 & $0.021^{*}$ \\
\hline MF Phase $\times$ Shot & 26.136 & 1.149 & 0.227 & $0.035^{*}$ \\
\hline CCI Group & 2 & 21.639 & $\leq 0.001^{*}$ & 0.267 \\
\hline CCI Phase & 4 & 1.358 & 0.252 & $0.042^{*}$ \\
\hline CCI Shot & 7.652 & 0.635 & 0.742 & 0.005 \\
\hline CCI Group $\times$ Phase & 8 & 378.753 & 0.936 & $0.0023^{*}$ \\
\hline CCI Group $\times$ Shot & 15.3 & 1.548 & 0.08 & $0.024^{*}$ \\
\hline CCI Phase $\times$ Shot & 30.606 & 2.007 & $0.001^{*}$ & 0.060 \\
\hline
\end{tabular}

CCI: Co-contraction index, Df: Degree of freedom, MF: Multifidus, TA: Transverse Abdominis. * - Level of significance was set at 0.05 (2-tailed).

Table 4. Post-hoc analysis between groups and phases

\begin{tabular}{|c|c|c|c|c|}
\hline \multicolumn{2}{|c|}{ Variables } & TA & MF & CCl \\
\hline \multirow{4}{*}{ Groups } & Group A - Group B & $\leq 0.001^{*}$ & $\leq 0.001^{*}$ & $\leq 0.001^{*}$ \\
\cline { 2 - 5 } & Group B - Group C & $\leq 0.001^{*}$ & 0.089 & 0.294 \\
\cline { 2 - 5 } & Group A - Group C & $\leq 0.001^{*}$ & $\leq 0.001^{*}$ & $\leq 0.001^{*}$ \\
\hline Phases & Pre-Draw - Draw & 1.000 & 1.000 & 1.000 \\
\cline { 2 - 5 } & Draw - Anchor & 0.180 & 1.000 & 0.241 \\
\cline { 2 - 5 } & Anchor - Release & $0.001^{*}$ & 1.000 & 1.000 \\
\cline { 2 - 5 } & Release - Follow Through & 0.911 & 1.000 & 1.000 \\
\hline
\end{tabular}

CCI: Co-contraction index, MF: Multifidus, TA: Transverse Abdominis. * - Correlation is significant at the level of 0.05 (2-tailed). 


\section{Discussion}

The major purpose of the study was to quantitatively measure the role and evaluate the activation of the core muscles (TA and MF) during different phases of archery among athletes having different levels of training age. Our study showed the highest activity in the elite group for the concerned muscles and their CCI. Elite archers tend to use more of their core muscles, which was evident from \%MVIC in all shots during every phase. The findings can be concluded as elite archers stabilize their body with CCI of the core muscles to optimally concentrate on their distal movements such as those of forearm muscles, which in turn can be related to their enhanced level of performance and skill in the sport $[7,8]$. This strategy is almost totally different in the beginner and trained archers where they use less of the co-activation which could be interfering with their upper limb movements essential for accuracy and consistency of the shots [6]. Many researchers believe this higher activity could be related to the training effects and neuromuscular adaptation of the archers. The trained and the beginner group showed a similar pattern of activation but the level of activity recorded was lesser, which may lead us to suggest dynamic core muscle training in elite group $[5,19]$.

Our results also showed that elite and beginner groups had different TA and MF activity and CCI; however, between elite and trained groups, only TA activity was found to be significantly different, which indicated that the trained group was similar to the elite group in MF activity and CCI, which might have resulted from the shot distance used in this study. We have noted the importance of TA during the stance phase, which can be correlated to the findings of this study [20]. While MF activity and CCI are similar in both the concerned groups, TA could be a determining factor for higher skill and accuracy in the sport. A significant difference between trained archers and beginners in TA, MF activity and CCI provided a clear picture of the training effects on the concerned muscles. The elite and the beginner groups were different in the level of expertise and performance in the sport, which relates with our SEMG findings. The results of our study revealed a significant difference between phase analysis of TA activity; however, no significant difference was noted in MF activity and CCI. The findings of MF activity and CCI can be concluded as they remain active all throughout the different phases of the shot, while that of TA changes during different phases of the shot, which may be predisposed to its functional and biomechanical implications. TA activity changes all throughout the phases and it has been reported to have a pivotal role in the stance phase which is preceded by the predraw phase, where we can see the initiation of the activity of the distal muscles. The spine acts as the base of stability in the dynamic draw phase till the follow through phase. Being a spinal stabilizer, MF becomes active from the pre-draw phase and minimally changes over the preceding phases, while the infero-medially situated TA changes in activity with the movement of the trunk and the rib cage. During the release phase, a large amount of kinetic energy dispersion is seen, which relates to the short burst of activity seen in the s EMG. The CCI did not change throughout the different phases, which can be attributed to static activity of the MF. The between-phase results showed a significant difference only in the anchor phase followed by the release phase in TA activity. Anchoring is achieved with minimally visible movement comprising of slight movements in the scapulo-humeral complex and some postural adjustments, which are the only dynamic components of this phase which is preceded by aiming and release; thus, the decrease in activity of the muscles during anchor can be understood.

Release is the phase where the stored energy is being translated and directed towards the target. The sudden burst of activity after a much static anchor phase could be seen as one of the possible reasons which might relate to our findings. The reaction force of release is countered by the activation of the postural muscles. Interplay of forces and muscle activity are best seen in the phases of anchor and release and hence the difference in activity. The evaluation of the between-phase effect was done according to their relative occurrence in the sport and this might have contributed to the results. No significant difference between the shots was observed, which could be attributed to the same technique used in every shot by all the archers irrespective of any group. Moreover, we assessed only ten shots, which might have interfered with the findings of the study. The external variables like wind velocity, temperature, time of the day etc. play an important role in performance of the shot, and all these factors were kept in control within the laboratory procedure. A significant difference in the interaction between phase $\times$ shot was observed for CCI only, which might be attributed to the dynamic fluid nature of the shots, which correlates to our interpretation of activity changing during the phases in every shot.

The findings of this study relate with earlier studies which have concluded the role of core muscles in stability and its implications on distal mobility $[19,7]$ and adaptation of a stable posture in which there is efficient use of forces and their interplay with the biomechanics of the body. An early study does contradict our findings but this study involved only two archers and thus the findings should be considered more specific than general [21].

The results have an on-field application on the activation of core muscles during archery and might have an important implication on the inclusion of core muscle exercises in the training protocol of archers. The study indicates that co-activation of postural muscles gives a stabilized base, which helps in better performance of the appendicular movements needed for a better shot. Also, beginners must undergo a specific core dynamic stabilization exercise initially for improvement in their archery performance. The present study had a few limitations. Firstly, the level of fitness of the archers might have confounded the results to some level. Secondly, the shot length might have some implications on the results and the findings might differ with different shot lengths. Lastly, the study undertook only ten shots, which might have contributed to a difference in the results.

There is a lack of awareness around the pivotal concept of stabilization during archery, which translates into incompetent evaluation and diagnosis. Further studies focusing on various distances of shot lengths of $30 \mathrm{~m}, 70 \mathrm{~m}$ etc. and large diverse population including females and older athletes must be included in the study. Also, core muscle interventions with a control group as well as different techniques and forms of archery with a higher number of shots should be implemented to better understand their effects on the performance of archers.

\section{Conclusion}

It has been concluded with the findings of the study that the elite archers showed more activity of the core muscles (TA and $\mathrm{MF}$ ) and their CCI was found to be much higher with respect to the trained and beginner archers. Moreover, we reported a change in the co-activation of the muscles between the interactions of the phase and the shot, which supports our hypothe- 
sis over the role of core muscles in archery. Therefore, it is essential for an archer to understand the interplay of stabilization and dynamicity of the core and incorporate dynamic stabilization exercises into their training.

\section{Acknowledgments}

The authors would like to thank the University for providing the necessary support for carrying out the research.

\section{References}

1. Simsek D., Cerrah A.O., Ertan H., Soylu R.A. (2018). Muscular coordination of movements associated with arrow release in archery. South African Journal for Research in Sport, Physical Education E Recreation 40(1).

2. Oliver G.D., Washington J.K., Barfield J.W., Gascon S.S., Gilmer G. (2018). Quantitative analysis of proximal and distal kinetic chain musculature during dynamic exercises. The Journal of Strength \& Conditioning Research 32(6), 15451553. DOI: 10.1519/JSC.0000000000002036

3. Kibler W.B., Press J., Sciascia A. (2006). The role of core stability in athletic function. Sports Medicine 36(3), 189-198. DOI: 10.2165/00007256-200636030-00001

4. Landers D.M., Boutcher S.H., Wang M.Q. (1986). A psychobiological study of archery performance. Research Quarterly for Exercise and Sport 57(3), 236-244. DOI: 10.1080/02701367.1986.10605403

5. Ertan H., Kentel B., Tümer S.T., Korkusuz F. (2003). Activation patterns in forearm muscles during archery shooting. Human Movement Science 22(1), 37-45. DOI: 10.1016/s01679457(02)00176-8

6. Nishizono H., Shibayama H., Izuta T., Saito K. (1987). Analysis of archery shooting techniques by means of electromyography. In Tsarouchas L., Terauds J., Gowitzke B.A., Holt L.E. (eds.) ISBS-Conference Proceedings Archive (pp. 364372). Athens: Greece.

7. Shinohara H., Urabe Y. (2017). Analysis of muscular activity in archery: a comparison of skill level. The Journal of Sports Medicine and Physical Fitness 58(12), 1752-1758. DOI: 10.23736/S0022-4707.17.07826-4

8. Tinazci C. (2011). Shooting dynamics in archery: A multidimensional analysis from drawing to releasing in male archers. Procedia Engineering 13, 290-296. DOI: 10.1016/j. proeng.2011.05.087

9. Ertan H. (2009). Muscular activation patterns of the bow arm in recurve archery. Journal of Science and Medicine in Sport 12(3), 357-360. DOI: 10.1016/j.jsams.2008.01.003

10. Musa R.M., Abdullah M.R., Maliki A.B.H.M., Kosni N.A., Haque M. (2016). The application of principal components analysis to recognize essential physical fitness components among youth development archers of Terengganu, Malaysia. Indian Journal of Science and Technology 9(44), 1-6.

11. De Oliveira I.O., Pilz B., Junior R.L.S., Vasconcelos R.A., Mello W., Grossi D.B. (2018). Reference values and reliability for lumbopelvic strength and endurance in asymptomatic subjects. Brazilian Journal of Physical Therapy 22(1), 33-41. DOI : 10.1016/j.bjpt.2017.09.008

12. Hodges P.W., Gandevia S.C., Richardson C.A. (1997). Contractions of specific abdominal muscles in postural tasks are affected by respiratory maneuvers. Journal of Applied Physiology 83(3), 753-760. DOI: 10.1152/jappl.1997.83.3.753

13. Ng J.K., Kippers V., Richardson C.A. (1998). Muscle fibre orientation of abdominal muscles and suggested surface
EMG electrode positions. Electromyography and Clinical Neurophysiology 38(1), 51-58.

14. Plamondon A., Serresse O., Boy, K., Ladouceur D., Desjardins P. (2002). Estimated moments at L5/S1 level and muscular activation of back extensors for six prone back extension exercises in healthy individuals. Scandinavian Journal of Medicine E Science in Sports 12(2), 81-89. DOI: 10.1034/j.1600-0838.2002.120204.x

15. Arokoski J.P., Kankaanpää M., Valta T., Juvonen I., Partanen J. et al. (1999). Back and hip extensor muscle function during therapeutic exercises. Archives of Physical Medicine and Rehabilitation 80(7), 842-850. DOI: 10.1016/s0003-9993(99)90237-x

16. Hodges P.W., Bui B.H. (1996). A comparison of computer-based methods for the determination of onset of muscle contraction using electromyography. Electroencephalography and Clinical Neurophysiology 101(6), 511-519. DOI: 10.1016/s0013-4694(96)95190-5

17. Rudolph K.S., Axe M.J., Buchanan T.S., Scholz J.P., Snyder-Mackler L. (2001). Dynamic stability in the anterior cruciate ligament deficient knee. Knee Surgery, Sports Traumatology, Arthroscopy 9(2), 62-71. DOI: 10.1007/s001670000166

18. Ertan H., Soylu A.R., Korkusuz F. (2005). Quantification the relationship between FITA scores and EMG skill indexes in archery. Journal of Electromyography and Kinesiology 15(2), 222-227. DOI: 10.1016/j.jelekin.2004.08.004

19. Metcalf H.G. (1955). Field archery is fun. Journal of Health, Physical Education, Recreation 26(6), 10-11.

20. Hennessy M.P., Parker A.W. (1990). Electromyography of arrow release in archery. Electromyography and Clinical Neurophysiology 30(1), 7-17.

Submitted: March 22, 2021

Accepted: April 2, 2021 